\title{
Sentinel Node and Ovarian Tumors: a Series of 20 Patients
}

Reita H. Nyberg, M.D., Department of OB\&GYN, Tampere University Hospital, Pirkanmaa Hospital District, Tampere, Finland

Pasi Korkola, Lic.Tech., Department of Nuclear Medicine, Medical Imaging Centre, Pirkanmaa Hospital District, Tampere, Finland

Johanna U. Mäenpää, Prof., Department of OB\&GYN, Tampere University Hospital, Pirkanmaa Hospital District, Tampere, Finland; School of Medicine, University of Tampere, Tampere, Finland

Corresponding Author and Reprints:

Dr. Reita Nyberg

P.O.Box 2000, 33521 Tampere, Finland

fax: +358331164396

telephone: +3583503847500

reita.nyberg@pshp.fi

This is a peer-reviewed manuscript. The original article has been published in International Journal of Gynecological Cancer. 2017, 27(4), pp. 684-689. http://dx.doi.org/10.1097/IGC.0000000000000948.

We declare having no commercial interest. We have not received any material support for this study. Reita Nyberg and Johanna Mäenpää have received a research grant from Cancer Foundation (Cancer Society of Finland). 


\section{Abstract}

Objective: Intraoperative detection of ovarian sentinel nodes has been shown to be feasible. We examined the detection rate and locations of sentinel nodes in patients with ovarian tumors. We also aimed to assess the reliability of sentinel node method in predicting regional lymph node metastasis.

Methods: Twenty patients scheduled for laparotomy due to a pelvic mass were recruited to the study. In the beginning of the laparotomy, radioisotope and blue dye were injected under the serosa next to the junction of the ovarian tumor and suspensory ligament. The number and locations of the hot and/or blue nodes/spots were recorded during the operation. If the tumor was malignant according to the frozen section, systematic lymphadenectomies were performed, the sentinel nodes sampled separately and their status compared to other regional lymph nodes.

Results: Eleven patients had a right-sided ovarian tumor, 7 patients a left-sided tumor and 2 patients bilateral tumors. A median of 2 sentinel nodes/locations per patient (range 1-3) were found. Sixty percent of all SNs were located in paraaortic region only, compared to $30 \%$ in both paraaortic and pelvic areas and $10 \%$ in pelvic area only. Both uni- and bilateral locations were found. In $83 \%$ of the cases with more than one sentinel node location, they were located in separate anatomical regions. In three patients, systematic lymphadenectomies were performed. One of them had nodal metastases in two regions and also a metastasis in one of her two sentinel nodes in one of those regions.

Conclusions: In patients with ovarian tumor(s), the detection of sentinel nodes is feasible. They are located in different anatomic areas both ipsi- and contralaterally, although most of them are found in the paraaortic region. The reliability of the sentinel node concept should be evaluated in the framework of a multicenter trial.

Key words: sentinel lymph node; ovarian tumor; early ovarian cancer; lymphadenectomy; surgical staging 


\section{Introduction}

Pelvic and paraaortic lymphadenectomy is considered to be a mandatory part of surgical care in early ovarian cancer. Accurate surgical staging is associated with better prognosis, provided that at least 10 nodes are harvested from different and specific retroperitoneal sites; especially paraaortic/paracaval nodes between the inferior mesenteric artery and the renal vein, superficial iliac nodes and nodes from the obturator fossa (1). Morever, larger number of removed nodes increases the likelihood of finding more lymph node metastasis (2). On the other hand, the increasing extend of the lymphadenectomy carries increasing risk of of serious complications (1), the blood loss and proportion of patients transfused, the duration of the surgery and the length of hospital stay (3).

During the surge of mini-invasive surgery in gynecological cancer, sentinel node (SN) concept has been adopted into surgery of early vulvar, cervical and endometrial cancer (4). In early ovarian cancer (EOC), the concept has been mainly overlooked because of a laborious reachability of the intra-abdominal injection site, a presumption of the slowness of commonly used tracers and a fear of spillage of tumor cells following the injection.

In 2011, Nyberg et al. reported a successful intraoperative use of blue dye and technetium isotope in detection of ovarian SNs after tracer injection into a normal postmenopausal ovary in patients with highrisk uterine cancer. The SN detection rate during systematic lymphadenectomies in a series of 16 patients was $94 \%$, and all SNs were located in the paraaortic regions (5). Three years later, Kleppe et al. described that the use of blue dye and radioisotope in ovarian tumor patients was successful in finding SN locations in all of their 21 patients. They injected the tracers into two sites in ovarian ligaments outside the suspicious tumor to prevent any potential dissemination of cancer cells. After injection, they located the hot SN sites transperitoneally with a gamma detector and retroperitoneally, if the tumors proved to be malignant and lymphadenectomies were carried out (6).

With our patient series, we wanted to examine the detection rate and locations of SNs by conventional tracers (blue dye and radioisotope) in patients with suspicious ovarian masses. We also aimed to evaluate the reliability of SN method in predicting regional lymph node metastasis, in case the mass proved to be malignant in frozen section analysis.

\section{Materials and Methods}

From December 2010 through September 2013, 20 eligible women with either unilateral or bilateral ovarian masses were recruited to the study. The inclusion criteria were as follows: scheduled open surgery (total abdominal hysterectomy TAH and bilateral salpingo-oophorectomy BSO) to remove the suspicious mass(es), which was estimated to involve the ovary/adnex only. In premenopausal women with intact uterus, also a negative pregnancy test within 24 hours before surgery was required. The exclusion criteria were as follows: previous allergic reaction to blue dye or human albumin, and signs of malignant spread to the abdominal cavity in preoperative imaging. Ascites alone and/or elevated serum CA 125 without any other sign of dissemination were not exclusive. 
The patient demographics, previous operative history and the largest diameter of the ovarian tumor(s) from each patient were recorded.

In the beginning of each laparotomy, the adnexal mass (or masses) was exposed. This sometimes required liberation of adhesions and/or lifting the large tumor outside the abdominal cavity. Then, $1 \mathrm{ml}$ of ${ }^{99 m}$ Technetium-labelled human albumin colloid (Nanocoll ${ }^{\circledR}$, GE Heathcare, Saluggia, Italy) was slowly injected to one spot under the serosa next to the lateral junction of the ovarian tumor (mesovarium) with a 27-gauge needle (Figure 1). The preparation of the radiopharmaceutical was performed in the Department of Nuclear Medicine on the same day. The needle was then kept in its place, the syringe changed and $2 \mathrm{ml}$ of patent blue dye (Bleu Patenté $\mathrm{V}^{\circledR}$, Guerbet, Paris, France) was injected to the same spot, to prevent the tracers to spill out from multiple needle holes and stain the operation field. In the case of bilateral tumors, the tracers were injected to both sides.

After a minimum of a 10-minute-interval, during which the abdominal cavity was examined, the peritoneal cytology taken and the passage of the blue dye noted, the adnexal mass or masses were removed, opened and sent to a pathologist for frozen section analysis. After that, the operation was continued with hysterectomy (unless previously removed). If the mass was benign or borderline not needing lymphadenectomies, the pelvic and paraaortic areas were then closely examined transperitoneally for the visible blue dye and extra radiation without opening the peritoneum or removing the nodes. A count of tast least $10 \mathrm{X}$ the background radiation was thought to represent a "hot" spot and SN location, using a hand-held gamma detector (Neo2000 ${ }^{\mathrm{TM}}$, Neoprobe, Dublin, Ohio). The number and locations of each blue and/or hot spots were marked on a map, that contained all significant lymphatic regions including upper paraaortic (PA) and paracaval (PC) area (above inferior mesenteric artery IMA), lower PA/PC area (below IMA), right and left common iliac area (upper pelvic area), right and left external iliac area and right and left obturator area (lower pelvic area), as well as the method of their detection. In the cas e of a borderline tumor, a complete surgical staging (CSS; peritoneal biopsies, omentectomy, appendicectomy) was then performed. If the tumor was malignant, systematic pelvic and paraaortic lymphadenectomies were carried out as a part of CSS, when technically possible. After opening the peritoneum, all retroperitoneally detected SNs (hot and/or blue) were sampled separately and their location marked on the map similarly as above.

The non-SNs were processed according to a standard protocol for lymph node examination; they were cut into single sections or, if more than $1 \mathrm{~cm}$ in diameter, into 2-3 sections and stained with hematoxylin and eosin (H\&E) before microscopy. The SNs were cut into $2 \mathrm{~mm}$ sections for H\&E staining. Ultrastaging and immunohistochemistry were not used. All detected metastases and their locations were recorded, and the status of each SN was compared to the status of the non-SNs in the same region.

Pearson's correlation coefficient was used to estimate the association between tumor size and SN number. A p-value less than 0.05 considered statistically significant.

The study protocol was approved by the local Ethics Committee of Pirkanmaa Hospital District (approval number R10072). A written informed consent was obtained from all patients.

\section{Results}

Twenty women, median age of 63.3 years (range 41.1-80.7 years) and BMI of 25.8 (range 21.5-36.1), were enrolled. The patient data is shown in Table 1. In women with previous open surgeries, a careful liberation 
of adhesions had to be made before the injection site was properly exposed. Two tumors were torquated (patients \#12,\#18) and the latter also buried under adhesions requiring liberation. The distribution of the tumor aspects is shown in Table 2. The median preoperative diameter of the right-sided tumors was $11 \mathrm{~cm}$ (range $5-20 \mathrm{~cm}$ ) and that of the left-sided tumors $7 \mathrm{~cm}$ (range $3.5-21 \mathrm{~cm}$ ). One right-sided tumor was found to be ruptured, and according to the symptoms of the patient, that had happened the night before surgery. Both tracers were successfully injected as described above to all 20 women, and no adverse or allergic reactions were noted. The median injected activity of Nanocoll ${ }^{\circledR}$ to one side was $20.0 \mathrm{MBq}$ (range 17.0-26.6 $\mathrm{MBq})$. The median interval between the tracer injections and the beginning of the removal of the tumor(s) was 12 minutes (range 10-33 minutes).

During the operation, eleven women had benign frozen sections, and the final histopathological examination confirmed those results. Four women had borderline tumors. In one case (patient \#17), the pathologist could not exclude invasion based on the frozen section. As there were also palpable prominent lymph nodes on the right paraaortic region, systematic lymphadenectomies were carried out and 3 hot and blue SNs were found and sampled on that area. The final diagnosis was serous borderline tumor, with endosalpingiosis present in two out of three SNs.

Five women had a malignant disease (patients \#7, \#8, \#15, \#16,\#20). During the examination of the abdominal cavity, an unexpected carcinosis was found in two of them (\#8 and \#16). Patient \#8 had a final diagnosis of serous ovarian cancer FIGO stage $3 \mathrm{a} 2$ with suboptimal surgical result, and \#16 had a breast cancer metastasis in the right ovary, spreading to the abdominal cavity. In both, SN locations (1 and 3, respectively) were transperitoneally detected and mapped. Patient \#20 had a BMI of 36.1, and because of technical difficulties, the lymphadenectomies were passed, but one hot SN location was found and mapped. Altogether, systematic lymphadenectomies with separate SN samplings were performed to 3 patients ( $15 \%$ of study population), and in the rest of the patients, a transperitoneal SN mapping was carried out.

We were able to locate 1-3 SNs (median of 2) from each woman, resulting in total of $36 \mathrm{SN}$ sites. For both right and left-sided tumors, the mean number of SNs was 2, with a range of 1-3 and 1-2, respectively. In the case of bilateral tumors, the median number of SNs was 2 (range 2-3). There was no correlation between the size of the tumor and the number of detected SN sites per patient $\left(r^{2}=0.0012\right.$, two-sided $\left.p=1.00\right)$. In 12 patients $(60 \%)$, more than one SN was found, and in 10 cases $(83 \%)$ they were located in the separate anatomical regions. The distribution of SN locations and laterality according to the tumor side is shown in Table 2. In eighteen patients with unilateral tumors, most of the SN sites were ipsilateral (83\%, 15 patients), and contralateral only in one patient (6\%), while bilateral SN sites were found in $11 \%$ (2 patients). With bilateral ovarian masses, all the SN sites were detected bilaterally.

The regional distributions of all SNs are shown in Figure 2. Seventy-eight percent of all SN sites (28/36) were located in the PA region. In 12/20 patients (60\%), it was the only region where SNs were detected. In $6(30 \%)$ of patients, both PA and pelvic SN locations were seen together. In only two patients (10\%), the SNs were found solely in pelvis.

The left-ovary-related SN sites seemed to be located higher than those related to the right ovary; $64 \%$ vs. $30 \%$ were located above the IMA, respectively. While $18 \%$ of the left-ovary-related SN sites were traced to the upper pelvic (common iliac) region, no SN sites were found in the lower pelvic area. Ten percent of the right-ovary-related SN sites were found in the upper pelvic region (common iliac region) and $10 \%$ in the 
lower pelvic region. The tracers seemed to cross the midline from left to right only above the IMA level, whereas crossover from right to left was observed in the upper pelvic, lower PA and upper PA regions.

The results of the three patients with systematic lymphadenectomies are shown in Table 3.

Eighteen of all SNs (50\%) were detected both visually and with gamma detector, 17 (47 \%) by means of radiation only and one ( $3 \%$ ) only visually. With transperitoneal mapping (28 SN locations), $43 \%$ (12 SNs) were found by combining dye and radiotracer, $54 \%$ (15 SNs) with radiation only and $4 \%$ (1 SN) with dye only. Eight SNs were collected in lymphadenectomised patients, $75 \%$ (6 SNs) with combined method and $25 \%$ ( 2 SNs) by using gamma detector. The median intervals between injections and identification of SNs were 56 minutes (28-126 minutes) transperitoneally and 129 minutes (49-180 minutes) retroperitoneally.

\section{Discussion}

With a detection rate of $100 \%$, our results confirm that blue dye and radioisotope are swiftly transported from the mesovarium to the regional lymph nodes, and that perioperative SN mapping is feasible in patients with ovarian tumors.

The distribution of SN sites in our study is in line with literature concerning lymph node metastases in apparent epithelial ovarian cancer (EOC). According to a review with more than 1200 EOC cases, para-aortic metastases alone were found in $50 \%$ of patients, both para-aortic and pelvic metastases in $30 \%$ of patients and pelvic metastases alone in $20 \%$ of patients. In case of a unilateral tumor, $56 \%$ of the metastases were ipsilateral, $21 \%$ contralateral and $41 \%$ bilateral (2).

In the present study, the left-ovary-related SNs were located either in the PA (71\%) or PA and upper pelvic (29\%) areas, whereas the right-ovary related SNs were located PA (64\%), PA and pelvic (18\%) or solely pelvic (18\%) areas, including also lower pelvis. The left-ovary-related SNs were more often found above the IMA level than the right-ovary-related SNs (64 vs. 30\%, respectively). With unilateral tumors, unilateral SNs were more common than bilateral SNs (89 vs. $11 \%$ ). Nyberg et al. reported $64 \%$ of the left-ovary-related SNs to be located above the IMA level, while $94 \%$ of the right-ovary-related SNs were located below the IMA level. In that series no bi- or contralateral SNs were found (5). Kleppe et al. reported most of their SN locations being traced to PA/PC region (67\%), as only $9 \%$ were traced to pelvis and $24 \%$ to both regions. Ninety-three percent of SN locations were ipsilateral. In their study, half of transperitoneally detected SN sites related to the left ovary were located in the ipsilateral upper PA area, compared to $45 \%$ of the SN sites related to the right ovary. All their removed SNs after left-sided injections were found high in the upper left PA area, but the SNs after right-sided injections were mainly located at the IMA level (6). This asymmetry, present in all three studies, may be a reflection of the asymmetrical drainage of the right and left ovarian veins.

It has been purported that the tracers should be injected to the both ovarian ligaments $(6,7)$. According to a recent paper, three lymphatic drainage pathways from the ovaries can be distinguished. The two main routes drain the ovaries via the suspensory ligament toward the PA/PC areas, and via the proper ligament of the ovaries toward the obturator fossa and the internal iliac artery. The third minor pathway drains the ovaries via the round ligament to the inguinal lymph nodes, but is probably present only in small percentage of people. This study did not find any connections between right and left ovarian drainage, nor did it explain the asymmetry of lymphatic networks between right and left ovary. It however described that a considerable number of lymphatic vessels are present in mesovarium along the entire length of the ovary 
(7). To our experience, the tracers leak out from the tiniest holes and smudge the operation field, hampering the visibility of blue-stained lymphatics and the use of gamma detector in pelvis. Our one-spot injection to the mesovarium gave similar SN distribution results compared to the study by Kleppe et al. (6), although they injected the tracers to both ovarian ligaments. Although one should be cautious in making firm conclusions based on small materials, it seems unnecessary to use multiple injection spots.

In present study, blue dye was observed transperitoneally in less than half of the cases. Retroperitoneally explored, $75 \%$ of the hot nodes were also blue-stained. In the study of Kleppe et al., blue staining was not recorded at all in transperitoneal mapping. In retroperitoneal exploration, they identified blue dye only in one third of the patients (6). The visibility of blue dye depends on the interval between the injection and mapping and the thickness of the tissue around the lymphatic vessels. To our experience, blue dye shows where to seek for the radiation. However, a lack of blue staining does not exclude the possibility of hot nodes. A new tracer indocyanine green is reported to have an improved tissue penetration compared to blue dye. Replacing blue dye with it might enhance the visibility. The results in endometrial and cervical cancer have certainly been promising $(8,9)$.

Only one patient in our series (\#15) had lymph node metastases; in PC and right pelvic area. Two SNs were found in the lower PC area, one of which was metastatic and predicted correctly the surgical stage of the patient. However, there were no SNs detected in the right pelvic area. In the series by Kleppe et al, also one patient had lymph node metastases in SNs and in other regional nodes. She, however, had a final diagnosis of ovarian and synchronous endometrial cancer, and it was not conclusive which cancer the metastases were related to (6). The number of patients studied is still too small to allow for any conclusions about the reliability of SN concept in ovarian cancer. When adding our results to the study of Kleppe et al., 41 ovarian tumor patients have been operated on, 9 lymphadenectomies have been performed and only two patients ( $5 \%$ of all) have had lymph node metastasis. Based on the above figures, to collect 20 EOC patients with nodal metastasis, one should include approximately 400 ovarian tumor patients. It is not easy to find suitable patients with suspicious ovarian tumors but no signs of dissemination. Two of our patients (10\%) had more advanced disease than was preoperatively assumed. For us, it took several years to gather a series even this large. Obviously only a multicenter study could solve this problem.

We conclude that perioperative one-spot injection of blue dye and technetium isotope is feasible in detecting sentinel nodes in patients with ovarian tumor(s). One to three SNs per patient can be found with this method; they are usually ipsilateral, but both bi- and contralateral SN are conceivable. Our results confirm that the main lymphatic drainage route from ovary is to PA region, the SNs of the left ovary being usually located higher than those of the right ovary. Also, the pelvic SNs related to the left ovary seem to be located higher in the pelvis than those related to the right ovary. The reliability of the SN concept in ovarian cancer and its clinical applications should be evaluated in a larger multicenter study.

\section{Acknowledgement}

The authors thank M.D., pathologist Marita Laurila, for the advice and assistance with the SN analysis. 


\section{References}

1. Trimbos JB. Lymphadenectomy in ovarian cancer: standard of care or unnecessary risk. Curr Opin Oncol 2011;23:507-511.

2. Kleppe M, Wang T, Van Gorp T, et al. Lymph node metastasis in stages I and II ovarian cancer: A review. Gynecol Oncol 2011;123:610-614.

3. Maggioni A, Benedetti Panici P, Dell'Anna T, et al. Randomised study of systematic lymphadenectomy in patients with epithelial ovarian cancer macroscopically confined to the pelvis. $\mathrm{Br} J$ Cancer 2006;95:699-704.

4. Cibula D, Oonk MHM, Abu-Rustum N. Sentinel lymph node biopsy in the management of gynecologic cancer. Curr Opin Obstet Gynecol 2015;27:66-72.

5. Nyberg RH, Korkola P, Mäenpää J. Ovarian sentinel node - is it feasible? Int J Gynecol Cancer 2011;21:568-572.

6. Kleppe M, Brans B, Van Gorp T, et al. The detection of sentinel nodes in ovarian cancer: a feasibility study. J Nucl Med 2014;55:1799-1804.

7. Kleppe M, Kraima AC, Kruitwagen RFPM, et al. Understanding lymphatic drainage pathways of the ovaries to predict sites for sentinel nodes in ovarian cancer. Int J Gynecol Cancer 2015;25: 1405-1414.

8. Handgraaf HJM, Verbeek FPR, Tummers QRJG, et al. Real-time near-infrared fluorescence guided surgery in gynecologic oncology: A review of the current state of the art. Gynecol Oncol 2014;135:606613.

9. Darin MC, Rodrigues Gómez-Hidalgo N, Westin SN, et al. Role of indocyanine green in sentinel node mapping in gynecologic cancer: Is fluorescence imaging the new standard? J Minim Invasive Gynecol 2016;23:186-193. 


\section{Figure legends}

Figure 1. The injection spot of the tracers to the mesovarium.

Figure 2. The distribution (percentages and numbers) of sentinel nodes (SN) in all anatomic regions according to the injection site: all SNs, right-ovary-related SNs, left-ovary-related SNs, SNs of both ovaries, respectively.

Table 1. The patient demographics. $\mathrm{BMI}=$ body mass index, $\mathrm{TAH}=$ total abdominal hysterectomy, $\mathrm{BSO}=$ bilateral salpingo-oophorectomy, SVA=supravaginal uterine amputation, $\mathrm{BS}=$ bilateral salpingectomy, $\mathrm{BO}=$ bilateral oophorectomy, $\mathrm{ADH}=$ liberation of adhesions, $\mathrm{LAE}=$ pelvic and para-aortic lymphadenectomies, $\mathrm{OM}=$ omentectomy, $\mathrm{APP}=$ appendicectomy, $\mathrm{BP}=$ peritoneal biopsies, $\mathrm{BOT}=$ borderline ovarian tumor, $\mathrm{VH}=$ vaginal hysterectomy.

Table 2. The distribution and laterality of sentinel node locations in 20 patients according to the side of the ovarian tumor. Tumor side was also the tracer injection site. $\mathrm{SN}=$ sentinel node.

Table 3. The histopathological results of the retroperitoneal SN sampling and systematic lymphadenectomy. $\mathrm{PA}=$ paraaortic, $\mathrm{SN}=$ sentinel node. 


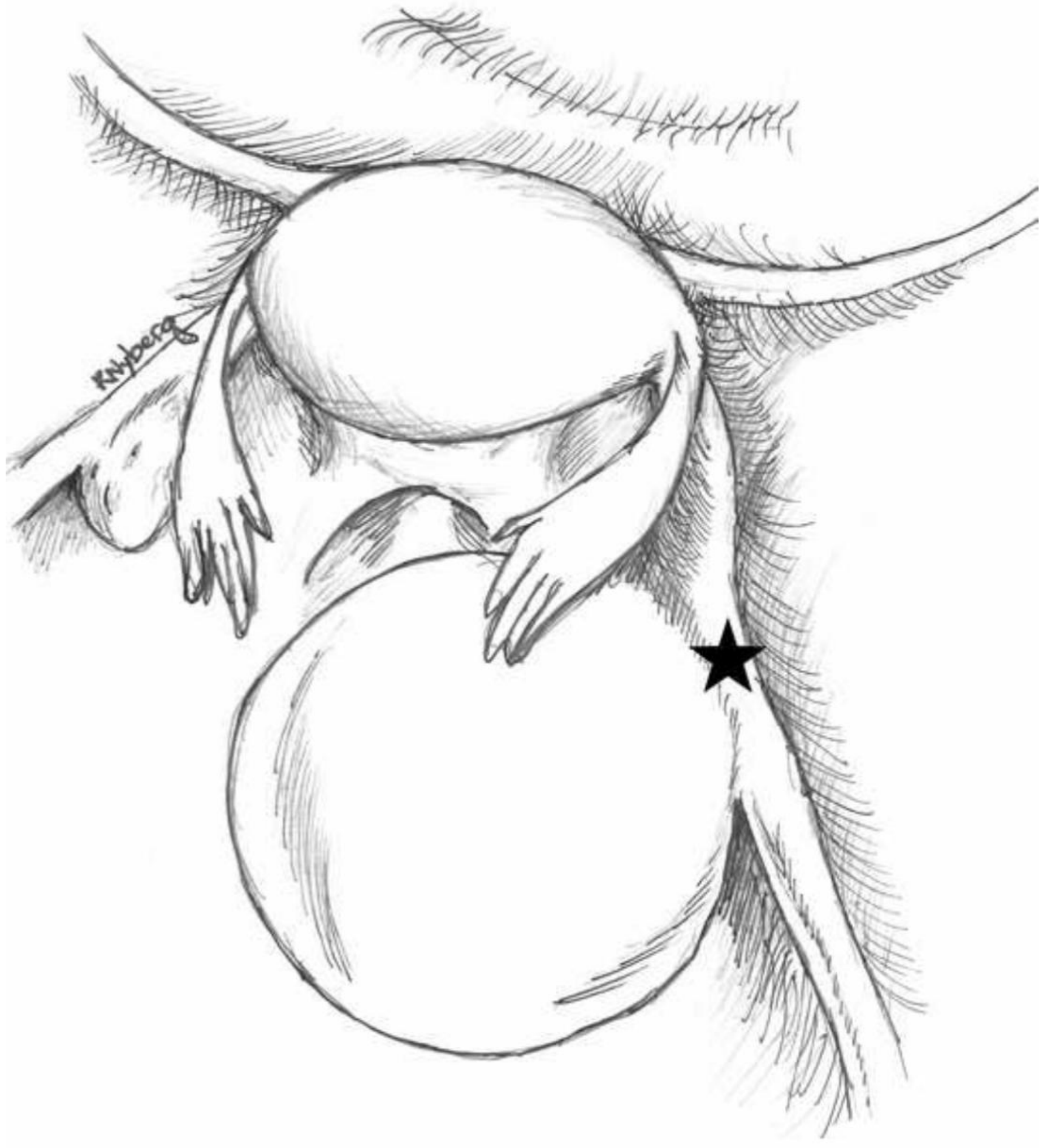




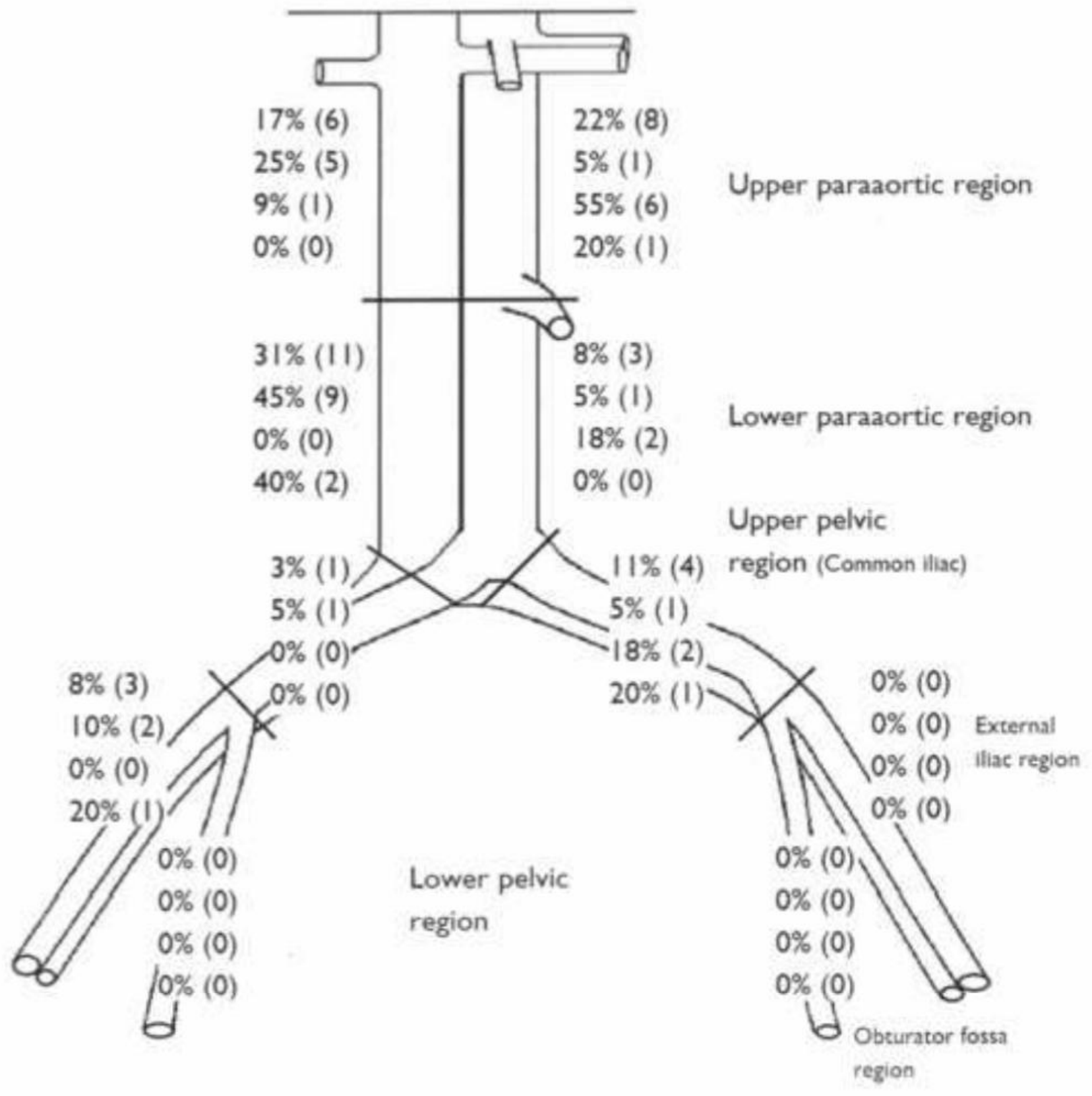




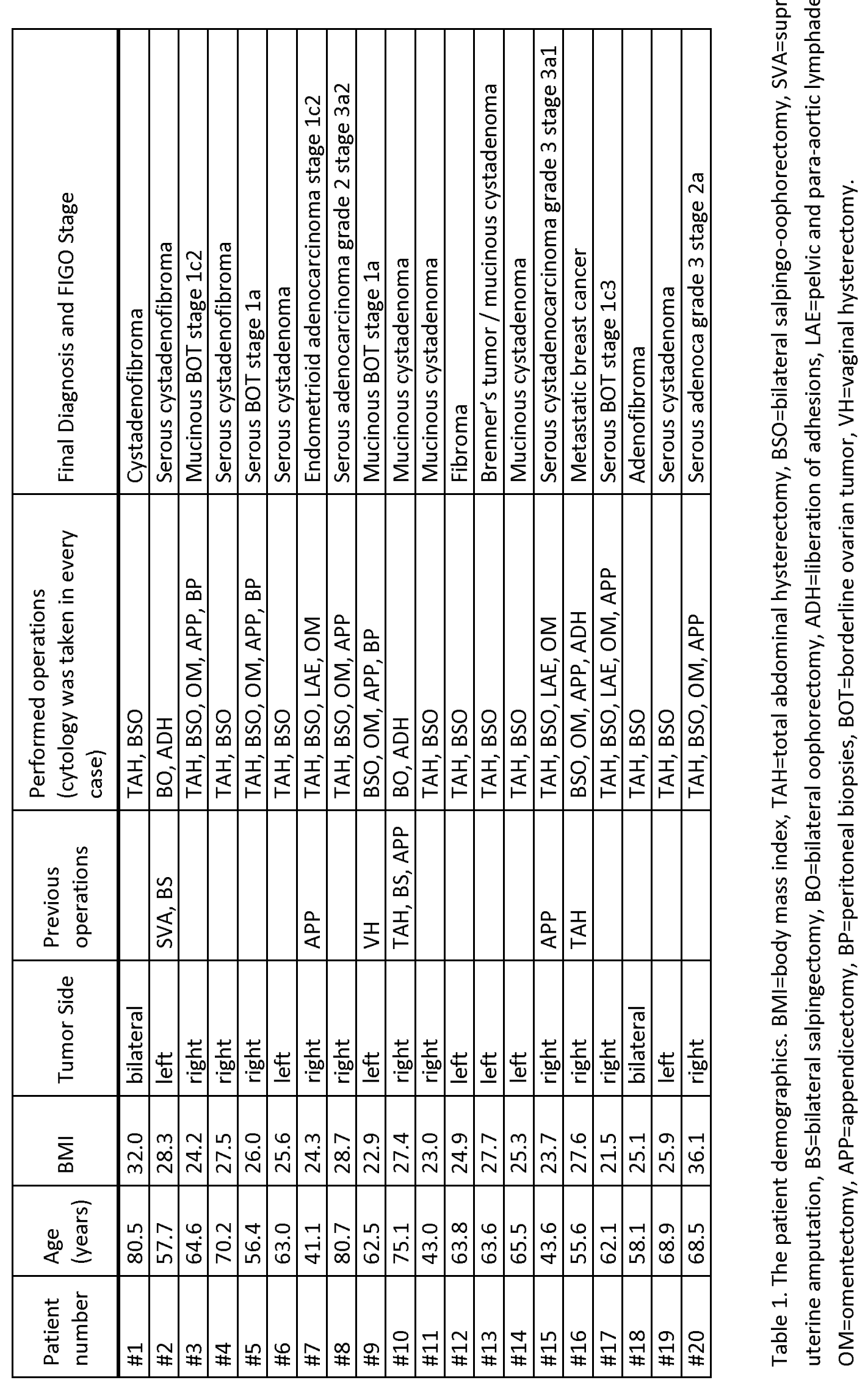




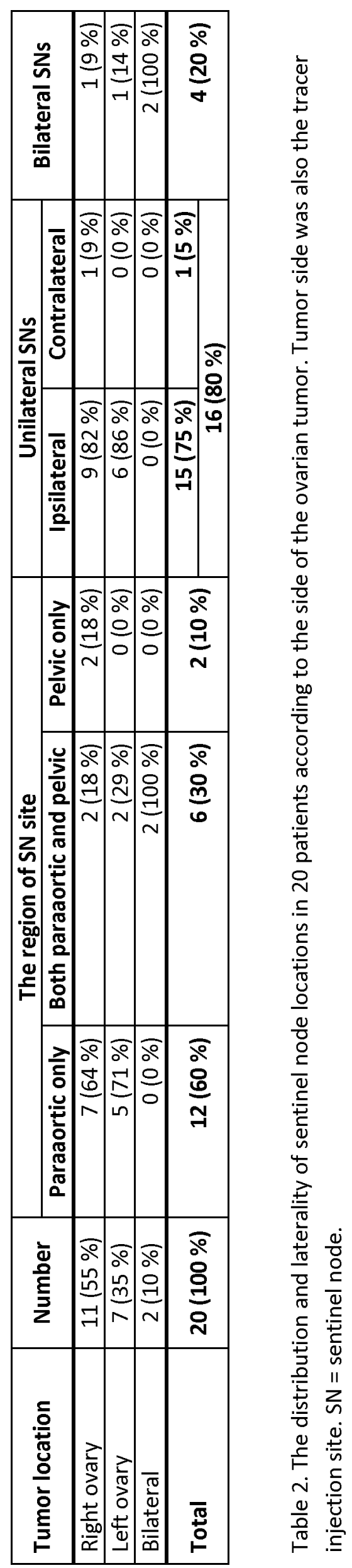




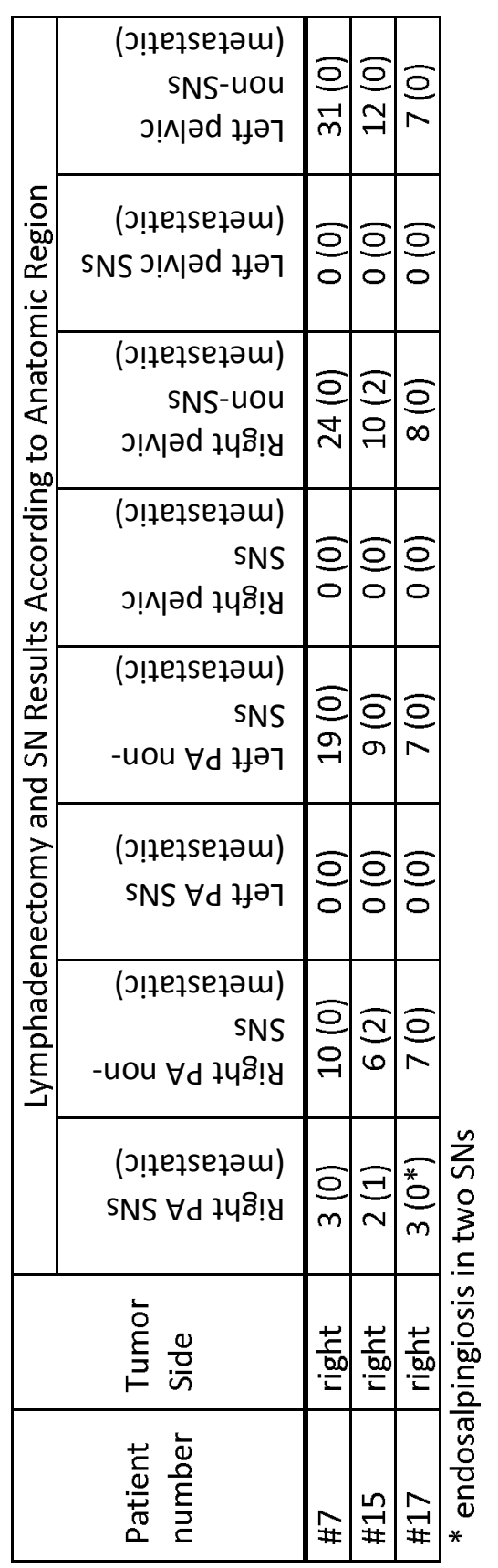

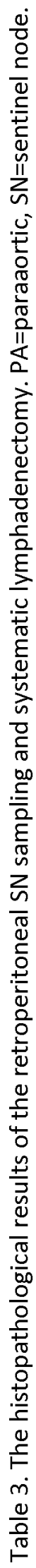

